\title{
Making Information Transparent as a Means to Close the Global Digital Divide
}

\author{
SORAJ HONGLADAROM \\ Department of Philosophy, Faculty of Arts, Chulalongkorn University, Bangkok 10330, Thailand; \\ E-mail: hsoraj@chula.ac.th
}

\begin{abstract}
This paper argues that information should be made transparent as a means to close the global digital divide problem. The usual conception of the digital divide as a bifurcation between the information 'rich' and 'poor' in fact does a poor job at describing the reality of the situation, which is characterized by multiple dimensions of digital divides in many contexts. Taking the lead from Albert Borgmann, it is recognized that the so-called information poor do possess a rich resource of information which needs to be fully accounted for in order to solve the global digital divide problem. In addition, making information transparent means, following the theory of development of Peruvian economist Hernando de Soto, that information should be made available and accessible through a system of representation. Floridi's account of information ethics thus needs to be supplemented by the realization that members of infosphere, which in his view consist of information objects, should be made transparent in order that the global digital divide be ameliorated. In other words there should be a system of information about information, namely semantic representation of objective information which enables the locals to make full use of their available informational resources.
\end{abstract}

\section{Introduction}

The digital divide - roughly the inequality of access to and benefiting from the new information and communication technologies - has generated a lot of debates and discussion in today's world. According to Toby Arquette, there has been an explosion of talks, conversations, reports, research works, media coverage, and so on about the issue, and the amount of talks about the issue is really staggering. ${ }^{1}$ It is quite clear that stories about the digital divide has a strong appeal to the public imagination. This is perhaps due to the fact that information and communication technologies (ICTs) have pervaded very deeply in the lives of an increasingly greater number of people, especially in the poorer countries.

In this paper, I would like to offer yet another perspective to this already well worn topic, perspective that ties it with the emerging field of philosophy of information, especially information ethics. More specifically, I will concentrate myself on the global digital divide - roughly, the disparity in access to the information and communication technologies between the developed and developing countries or societies. I will try to argue that a reason why the so-called information poor remain where they are is not because they lack information, but because, following Albert Borgmann (1999), they already possess a vast resource of information, albeit a different kind, and, following Hernando de Soto (2000), the rich resource of information available to them is locked up and cannot be fully utilized. Hence,

Minds and Machines 14: 85-99, 2004.

(C) 2004 Kluwer Academic Publishers. Printed in the Netherlands. 
Floridi's brand of information ethics that takes the infosphere as central to ethical concerns needs to be qualified. Floridi holds that the more information in the infosphere, the better off the world would become. I agree. However, the information in question would need to be made available to the public in general, and one should not regard the digital divide issue as a simple lack of information on one side and perhaps a glut of the same on the other. Instead the issue involves more on how the information that is already present with the so-called information poor is to be made available so that the flow of information is a two-way street rather than the one-way fare as usually conceived. Finding out how to solve this problem also involves tying information ethics with the also emerging field concerning deliberation on global justice, since one is now dealing with the problems emerging from the relations among cultures and nations of the world rather than from the members of one state only (O'Neill, 2000; Hongladarom, 2001).

\section{Characterizing the Digital Divide (or Divides)}

The gap between the so-called 'information rich' and 'information poor' is too broad to enable one to understand the complexities of what is currently going on because it presupposes that there is a simple divide between those who possess the technologies and enjoy their benefits and those who cannot do so for whatever reasons. Courtright and Robbin (2002) wrote that the term 'digital divide' itself is a metaphor evoking the image of a gulf, a chasm, between those who enjoy the technologies and those who do not. ${ }^{2}$ This presupposed bifurcation perhaps oversimplifies the issue and tends to lead one to think that the world is divided into two kinds of people, where in fact the reality is much more complex. ${ }^{3}$

Many scholars have also recognized that 'digital divide' is not a unitary concept that can be applied to the same type of phenomenon. On the contrary, one should more accurately talk about the digital divides, as there are many different kinds of the divide. Eszter Hargittai, for example, argues that there is the second-level digital divide, consisting of the gap between the skills people have when they are online (Hargittai, 2002). This is in contrast with the usual interpretation of the digital divide merely as the gap between those who possess or do not possess the technology. On the other hand, one can see that there are many types of the divide simply by looking at the situation in, say, the United States, where there is obviously a kind of divide and compare it to a country such as Thailand, where there is a divide too, but of a different kind. In the US, the divide can be seen quite clearly along racial lines, with a much higher percentage of whites enjoying the technology than blacks do. In Thailand, on the other hand, the divide is located along the urban and rural line, with urban, middle class people on one side and the rural farmers on the other. But even so this is still too crude a description of the actual conditions. It is clear that the difference here stems from, or could be explained by, the different historical paths that Thailand and the US took. But if that is the case, then talks of the digital divide as if it were a single phenomenon appearing all over the world would be too 
broad, and cannot do justice to the obvious role that cultures and historical paths play in policy deliberations on the digital divide.

Not only does the digital divide cut across many boundaries, it seems one could meaningfully talk about the divide among groups rather than among individuals. Floridi, on the other hand, argues that the digital divide is more a matter for individuals rather than groups or nations (Floridi, 2002b), since an interesting fact about it is that it can occur within the same family or within the same social context (Floridi, pers. comm.). In any case, it is apparent that there are wide disparities in the percentage points of those who are connected to the Internet in a developing country such as Thailand and the same type of number in, say, the US or Sweden. On average, a Thai would enjoy far less chance of being connected to the Internet than would an average American or Swede. ${ }^{4}$ Thus it seems one can talk about the divide among groups, as statisticians usually do when they compare percentage points of a type of phenomenon among various groups. Talking about the divide among groups is also essential when the focus is on global digital justice - a kind of global justice dealing with equal or unequal distribution of information use and connectedness through the information network across the globe.

Hence it is clear that the digital divide issue is part of the problem of global justice. In what follows I shall discuss the works of Luciano Floridi and Albert Borgmann. The aim is to show that a very important specific task for philosophy of information or in information ethics is to study how information is spread throughout the world as well as the role of culture in order to deliberate on the ethical issues involved.

\section{Information Ethics and Transparency of Information}

In a series of articles, Luciano Floridi advances a theory of information ethics that aims at establishing a moral theory based on the concept of information (Floridi, 1999, 2001, 2002a, b, 2003; Floridi and Sanders, 1999, 2002). For Floridi, information ethics is to be a kind of ethics that takes the collected sum of information itself, or the 'infosphere', as central. Standard ethical theories, such as Kantianism and utilitarianism, are based on the recognition that it is human beings that are to be the center of ethical deliberations. For Kantianism, as is well known, it is the reasoning capacity, and the recognition that human beings are to be treated as ends and never as means, that serves as the linchpin of the system. On the other hand, utilitarianism is based on the concept of the utilities, which are held as those of human beings. Floridi calls these ethical systems 'biocentric' ethics since they are focused on the biological creature. On the contrary, Floridi advances another kind of ethics, one that takes the minimalist position in that it focuses on the minimal condition of possibility of moral discourse itself. And since the environment, such as the infosphere itself and not just human beings, can be an object of ethical deliberation with no human interests involved, the concern of Floridi's theory is thus broader than the biosphere and the theory takes the infosphere itself to be central. The 
theory should not be interpreted as a lack of concern for human beings. Instead it argues that the concerns of a moral theory should be broader than merely with human beings only (Floridi, pers. comm.). Floridi wrote:

Biocentric ethics usually ground their analyses of the moral standing of bioentities and ecological systems on the intrinsic worthiness of life and the intrinsically negative value of suffering. IE [information ethics] suggests that there is something even more elemental than life, namely being, understood as information; and something more fundamental than pain, namely entropy. According to IE, one should also evaluate the duty of any rational being in terms of contribution to the growth of the infosphere, and any process, action or event that negatively affects the whole infosphere - not just an information entity - as an increase in its level of entropy and hence an instance of evil. Without information there is no moral action, but in IE information moves from being a necessary prerequisite for any morally responsible action to being its primary object. The crucial importance of this radical change in perspective cannot be overestimated. Typical non-standard ethics can reach their high level of universalisation of the ethical discourse only thanks to their biocentric nature. However, this also means that even Bioethics and Environmental Ethics fail to achieve a level of complete impartiality, because they are still biased against what is inanimate, lifeless, intangible or abstract (even Land Ethics is biased against technology and artifacts, for example). From their perspective, only what is intuitively alive deserves to be considered as a proper centre of moral claims, no matter how minimal, so a whole universe escapes their attention. Now this is precisely the fundamental limit overcome by IE, which further lowers the necessary condition that needs to be satisfied, in order to qualify as a centre of moral concern, to the minimal common factor shared by any entity, namely its information state. And since any form of being is in any case also a coherent body of information, to say that IE is infocentric is tantamount to interpreting it, correctly, as an ontocentric theory. The ethical question asked by IE is: "What is good for an information entity and the infosphere in general?" The answer is provided by a minimalist theory of deserts: any information entity is recognised to be the centre of some basic ethical claims, which deserve recognition and should help to regulate the implementation of any information process involving it. Approval or disapproval of any information process is then based on how the latter affects the essence of the information entities it involves and, more generally, the whole infosphere, i.e. on how successful or unsuccessful it is in respecting the ethical claims attributable to the information entities involved, and hence in improving or impoverishing the infosphere. IE brings to ultimate completion the process of enlarging the concept of what may count as a centre of minimal moral concern, which now includes every information entity. This is why it can present itself as a non-standard, patient-oriented and ontocentric macroethics (Floridi and Sanders, 2002, pp. 19-20). 
The passage quoted above quite neatly sums up Floridi's view on information ethics. Any action that promotes the infosphere, in so far as information ethics is concerned, is to be judged as good, and any that threatens it is to be judged the opposite. In other words, action that harms the infosphere, other things being equal, would be worse than action that promotes it, or leaves it untouched (Floridi, pers. comm.). ${ }^{5}$ Hence, Floridi's theory is a kind of naturalism in ethics, where normative evaluation of an action is to be based on whether that action promotes or demotes some kind of natural entity. One might well compare Floridi's theory here with Spinoza's. In any case, taking information as the center of ethical consideration may prima facie provide ammunition for arguing that the digital divide is a problem that needs to be solved. However, when one realizes, as I shall show presently, that there are many kinds of information and the so-called information poor are not actually as poor in information as one might think, and that the information that can break the digital divide needs to be transparent, the kind of information ethics proposed by Floridi here may not, as it stands, be adequate to the task.

In order to see how the last sentence of the previous paragraph is the case, let us take a brief detour and have a look at what Albert Borgmann has to say on the nature of information. In Holding on to Reality: The Nature of Information at the Turn of the Millennium (Borgmann, 1999) Borgmann advances an idea of information as being connected with human history and level of sophistication in their dealing with the natural environment. Borgmann sees that information, especially what he calls 'technological information', the kind of information that is made possible by the contemporary computer and communication technologies, is poised to replace reality altogether. And he is calling for a balance among, in his terms, 'natural', 'cultural' and 'technological' information.

According to Borgmann, natural information is the most primitive kind of information. The term 'primitive' here is used in the sense of something being simple and natural, not in the pejorative sense. However, it is clear that for Borgmann natural information stands first in the history of the development of information. Natural information is information about reality; it is itself part of the natural world that stands for another part. Thus the existence of round pebbles on the shore signals that a river is nearby, or the presence of such and such bird songs signals that such and such kind of birds are within hearing range. Black clouds in the sky show that rain is imminent, and so on. Natural information is the simple signaling by one part of nature to another part, mostly through physical cause and effect relations. Pebbles got rounded because of the water in the stream, and so the roundness of the pebbles signals the running of the water. Moreover, the information encoded within texts is also a kind of natural information. Texts can record what is happening far more accurately the memories.

Cultural information, on the other hand, is information for reality, since it contains recipes for designing reality. Thus information contained in cookbooks as well as architects' drawings are of this kind. Cultural information does not merely describes reality; it makes reality happen through its role as the blueprint, so to 
speak, for the designing and making of aspects of physical reality. In this sense, all music scores contain cultural information since they prescribe how a piece of music is going to sound like. The third kind of information, technological information, is information as reality. As technology progresses, so does its capacity for imitating and in the end replacing reality itself. Borgmann's example is the compact disc. The information contained in an audio CD, for example Bach's cantatas, is not only an imitation of the live music when the cantatas are performed, but the music one listens to on the $\mathrm{CD}$ threatens to become the real embodiment of the music itself. The accuracy and clarity of the music one hears on the $\mathrm{CD}$ is such that live performances become pale by comparison. Technological information can take us further away from reality through its power of becoming reality in itself. Virtual reality simulations, when perfected, are capable of creating an alternative reality where the old reality one can feel through one's immediate senses (not through the mediation of the technology) can seem 'unreal'. Borgmann, in short, is calling for us to hold back and take a reflective look at the current situation where technological information is threatening to take the primitive reality away from us, and to strike a healthy balance among the three types of information.

What this analysis of information in Borgmann implies for the digital divide problem is that it helps us become aware that talks about the divide presuppose that one is taking a one-sided view on technology and its progress. Having no technology, being unconnected to the World Wide Web and the other paraphernalia of communication technologies, has become a problem calling for a solution. The talks on the 'information rich' and the 'information poor' also presuppose that there is something, information, whose possession indicates whether one is rich or poor. Presumably the more information one possesses, the richer one becomes, and vice versa. ${ }^{6}$ However, Borgmann shows that there are more kinds of information than the technological one that seems to be implied by the talks about the digital divide. The poor villagers in remote areas in Thailand certainly do not lack natural information. In fact they are very rich in this regard. They have a rich set of resources that enable them to cope with the environment and potentially even to prosper. Knowledge and skills involved in planting rice, for example, involve a huge amount of natural information - knowing when to plant the rice, which seeds to sow, what are the proper techniques of tending the young rice, how to recognize potential pests and how to deal with them, when to harvest, and so on and on. Thus the fact that the farmers are unconnected to the Internet should not be taken to mean that they are information poor; in fact for a specific kind of information they are very rich. Urban blue-collar workers also can be quite rich in formation, although they don't own computers or do not know how to surf the Internet. What they are rich in includes how to negotiate the difficult tasks of living in the urban area, how to do their job and make a living, and so on. They might be involved with natural information, and in some cases they also deal with cultural information, as when they have to work out a plan or follow a set of guidelines. 
Thus, talking about a divide between the information rich and poor does not seem to be valid when one realizes that there are many kinds of information as Borgmann suggests. Those who are generally taken to be poor in information can be quite rich in other kinds, as we have seen. And those who are believed to be rich in information can indeed be rather poor in other kinds of information too. University professors who routinely check e-mails many times a day and are well versed in finding information through the search engines may be at a loss when asked to tell a strain of rice from another, something that a farmer can do with ease. The gap between the so-called information rich and information poor, then, is not a simple matter of one side not having enough information and the other having perhaps too much, but one has to bear in mind what kind of information too. The rural poor in Thailand generally possess a rich heritage of natural information which comprises their traditional knowledge bases; they also have a rich resource base in cultural information, stored in memories and texts. What they lack is perhaps the technological information, usually in digitized form stored in computer-accessible media. The existence of technological information presupposes computer networks, which in turn presuppose that there are resources for making the networks functional in the first place. But be that as it may, talks of the digital divide as if there were only one type of information, as mottoes like "the more information you have, the better off you will become" happen to illustrate, neglect the fact that there are the other kinds of information which are no less pertinent to individual and social life. Furthermore, as Eszter Hargittai (2002) has shown, merely being in possession of information does not automatically translate to one being on the right side of the digital divide. One has to factor in the skills involved in making full use of the resources at hand. Analogously, the 'rich' may be indeed quite poor when they do not know how to utilize the resources available to them.

Nonetheless, one cannot deny the fact that Thai farmers earn on average much less than their counterparts in the European Union and the US, and factory workers in Thailand enjoy far less leisure time and earn much less than university professors. Although there might be many kinds of information according to Borgmann, this does not drive away the fact of the digital divide (or divides). Thus something must be going on rather than the mere fact that one side of the divide enjoys more information than the other. Since the so-called information poor do in fact have quite a lot of information available to them (at least as natural and cultural information rather than technological one), what makes them on the wrong side of the digital divide can perhaps be explained by the fact that they either do not have the resources to make full use of what is available to them, or they are prohibited from doing so. The rural poor in Thailand, for example, are on the wrong side, not simply because they do not own computers nor because their villages do not have electric power or telephone lines - of course these are essential in bridging the divide - but also because they do not know how to translate whatever information available to them (in whatever form) into the kind of information that enables them to enjoy the full benefits that the information that is available to them is capable 
of bringing. The reason why the so-called information poor are likely to remain materially poor as well is not because they don't have the information that will take them out of poverty, but because the information they have remains implicit and thus unavailable to convert into capital, which can actually alleviate the poverty. The trick is to find how the store of information belonging to the so-called information poor could be made marketable and more valuable in economic terms. This requires some explanation, which I will do in the next section. Right now it is quite clear we have in our hands here a problem concerning justice, in fact global justice if we are talking about the global digital divide.

Now, however, we are in a better position to argue for the thesis that an ethics of the digital divide needs to take cultures and historical traditions into consideration. This is so because, as Borgmann argues, there are more kinds of information than the technological one, and the bifurcation of the people of the world into the information 'rich' and 'poor' is itself a poor way of defining the problem. However, Floridi's information ethics is predicated on the idea that ethical norms are based on the size of the infosphere - on whether the norms do improve or impoverish the infosphere. This works well if everything in the infosphere is transparent; that is, if any and all the information contained in the infosphere is readily available to be discerned and made use of by anybody who enters it. But if reality is such that the information remains locked up and thus unavailable to the poor villagers, then they remain poor, both informationally and economically, even though they may have a rich store of information. ${ }^{7}$ Whatever information the villagers have, such as the knowledge and skills involved in finding medicinal herbs in the forest and so on, cannot be converted into the kind of capital that can lift them out of poverty and ignorance because there is no mechanism to transfer these knowledge and skills in such a way that they are connected with the other existing knowledge and skill systems, thus becoming available to the global infosphere. In short, the information possessed by the villagers needs to be made transparent.

\section{The Mystery of Capital and How Information Is Represented}

In The Mystery of Capital: How Capitalism Triumphs in the West and Fails Everywhere Else, Hernando de Soto (2000) advances a thesis that the reason the West became successful mobilizing and making full use of capital is that the West has perfected the system of registration and accounting of resources, especially land, whereas the rest of the world is far behind in this regard. The basic idea is that it is information made transparent, i.e., made available through a system of representing, archiving and retrieving, that separates the West from the rest. The poor in the Third World countries, so argues de Soto, are in fact much richer than they seem because they possess, collectively, a vast store of information that has not been converted into the kind of information that can be, for example, digitized, catalogued and put to the legal framework. The poor may sit on a vast tract of 
land, but they do not legally own the land. They do not have land title deeds that can document their ownership. Without the title deeds, they have no way to create capital. Without any means to change the land into assets, they have no means by which they can create capital that can catapult them onto the world economy. The key issue here lies in how information is represented, or made transparent to the legal and economic system. Change how this information is perceived, and a vast amount of capital can be created.

For de Soto information plays a crucial role in his view of how property can be turned into capital. The process of representing the complex array of assets and property makes it 'mind friendly' (de Soto, 2000, pp. 218-221). Thus things very different from one another such as pieces of gold, tracts of land, pieces of jewelry, heads of cow can be made interchangeable and put to work under the same system. This is of course how the modern money system works. According to de Soto, "capital results from the ability of the West to use property systems to represent their resources in a virtual context. Only there can minds meet to identify and realize the meaning of assets for humankind" (de Soto, 2000, p. 218). The West captured the magic of turning base metals, gold, land, manufactured products, various types of service, and everything else into abstract numbers under one representational system that indicates their economic values. They developed a system whereby these values can be represented to the mind in such a way that the mind can work on them and not to the things directly. This is analogous to how the mind works in conceptual or language systems. Information needs to be made transparent and available through representational system; otherwise it would remain hidden and unaccounted for. Though in many cases it might be plainly visible, such as the pieces of land occupied by the poor in the Third World, without the representational system that enables the land to be documented and thus presented as a value in the system, it is as if the land is invisible, nonexistent.

De Soto's key idea is that representing something in a network of commensurable and fungible system gives it value and liquidity. A germ of this idea is from Searle's distinction between social and physical reality, where the former is constituted by a system of social relations and the latter by physical laws (Searle, 1995, pp. 1-4). Thus a piece of paper is a legal tender for 20 British pounds in social reality, and is a small slice of dead wood in the other. It is as if calling something by name elevates it into a member of a network of meaningfulness, a linguistic and conceptual network, and thus in a way creates its 'existence', in effect bringing part of physical reality to social reality. Philosophers have long been familiar with the important roles that conceptual system plays in thinking and cognition. It is well known that the debate between realism and anti-realism as regards to representational system focuses on whether the thing represented does have its separate existence apart from any means of representing it. ${ }^{8}$ Of course an economic argument is a far cry from a philosophical one, and one cannot rely on de Soto's argument here as an ally in arguing for an anti-realist position. Nevertheless, it is quite clear that information plays a crucial role in creating capital, which relies 
on a system of representation for it to work. And this information that can make the whole process work must not lie hidden and unaccounted for. In my words, it needs to be transparent.

Here is the connection between de Soto's view and the argument on the digital divide that I am trying to advance. We have seen that information must be transparent in order that those who possess it can benefit from it fully. In fact for a piece of information to be transparent is just for it to enter the representation system that gives it value in de Soto's sense. A tribe in a remote rain forest may possess a rich store of information, including some that could be life saving when developed into drugs, for example. The problem with the tribe, what makes it 'poor' in information, thus putting them on the wrong side of the digital divide, is that this store of information lies untapped and unrepresented. Well represented, the information could be of tremendous value not only in economic terms, but also on the humanitarian terms as the information would lead to life saving drugs. Floridi's infosphere should be populated only by those pieces of information that are well represented. That is, the pieces should be arranged in such a way that the mind finds it easy to work on them, such as through the cataloguing system used in libraries (but obviously this is not the only way). If this can be done, then a way can be found to solve the digital divide problem. The tribe can benefit from the potentially very lucrative pieces of information that they possess, and the world benefits from the life saving drugs that can be developed out of them.

\section{Information Sharing}

Floridi argues that entropy in information is an 'evil' and needs to be reduced or eliminated (Floridi, 1999; Floridi and Sanders, 1999). Simply put, entropy is the amount of loss of information when it is transmitted from one place to another, or when it is stored in a storage system that admits of some degree of loss. In order to bridge the digital divide, Floridi looks for actions that follow the following four basic norms of information ethics:

1. Information entropy ought not to be caused in the infosphere.

2. Information entropy ought to be prevented in the infosphere.

3. Information entropy ought to be removed from the infosphere.

4. Information ought to be promoted by extending, improving, enriching and opening the infosphere, that is by ensuring information quantity, quality, variety, security, ownership, privacy, pluralism and access (Floridi, 2002b, p. 4).

Thus Floridi aims at preventing entropy from entering or occurring in a system. Talks about entropy, however, seems at the face of it to convey the meaning that continual work has to be exerted on the infosphere to keep it running smoothly. The metaphor seems to be that of keeping a thermodynamic system working without 
increasing the entropy. This requires constant energy. Floridi, however, does not mean this usual physical sense when he used the term (Floridi, pers. comm.). Instead what he means by the term could be characterized simply by loss of information. Thus his use of the term is not metaphorical at all. Be that as it may, my contention is that a more appropriate metaphor seems to be a grid that can be laid down on the infosphere, making it possible to locate and triangulate everything inside. This means that, for the natural information possessed by the remote villagers in the Third World, a way needs to be found to categorize it in order that the information belongs to the infosphere in such a way that it can more substantially benefit those villagers.

What is needed here is that there should be a system of information about information, a kind of second-order information that deals with the natural and cultural information possessed by the villagers and made available to the world through cyberspace. Closing the digital gap does not only mean bringing in information from one presumably 'right' side of the gap to the supposedly 'wrong' one. Instead it means a more adequate, equitable and fair way of sharing information between the two sides. One needs to be careful that the so-called information poor, since they are less powerful than the other group, not be exploited by this way of sharing information and that they actually benefit from it. ${ }^{9}$ An evil that could emerge from this configuration if we were not careful is that the more powerful in cyberspace would exploit the second-order information obtained from the poor villagers without adequate compensation. This requires that there be justice in cyberspace which is global and does not limit itself to national boundaries only. There is unfortunately not enough space in this present paper to deal with this very important issue of justice in cyberspace, or information justice, but this could be a fruitful avenue of further research.

If the argument so far is on the right track, then 'information sharing' and 'making information transparent' should be the key concepts in solving the digital divide. Simply pouring in more information to the so-called information poor does not work. Neither does pumping money and other material resources to the poor and hoping that they somehow pick up on their own later on. Solving the digital divide problem can be accomplished if one realizes that the so-called information poor is in fact not poor at all. Instead they already possess a vast store of information which has enabled them to survive and even to prosper. Bridging the digital divide for them means bringing them over to the global economy, enlisting them as members of today's globalization. In some cases this might not be desirable, considering the various well known ill effects of globalization such as cultural or economic domination. However, it is not practicable now to shut oneself off from globalization. Thus a way needs to be found to combat those ill effects so that the villagers can enjoy the benefits of globalization. What lies in store for them is that they need to find a way to convert their already rich resources in natural and cultural information into something that can be converted into capital. If de Soto is right, which I believe he is, then the villagers' system of information should be 
made transparent through a mechanism that surveys and categorizes it in such a way that is 'mind friendly'.

The implication this has so far on information ethics is that the notion of the infosphere needs to be supplemented by that of transparency. The apparent optimism that anything that impoverishes the infosphere is bad and anything that improves it is good should be qualified. An action that concerns information process, other things being equal, is a good one in this case just in case it promotes the transparency of the information in the infosphere, and not good otherwise. ${ }^{10}$

\section{Notes}

${ }^{1}$ According to Toby Arquette, a sample of newspapers, journal articles, newswires, and similar mass media sources in the 11 year period from 1990 to 2000 reveals that "14,123 addressing the digital divide appeared. Even more revealing is the explosion in frequency in coverage of the digital divide issue: 1995, 3 stories; 1996, 31 stories, 1997, 43 stories; 1998, 316 stories; 1999, 2054 stories; 2000, 11,676 stories. This represents almost a $3000 \%$ increase in issue coverage since 1995. As of July 2001, 4,457 stories are devoted to the digital divide issue" (Arquette, 2002, p. 9).

${ }^{2}$ Courtright and Robbin (2002) have analyzed the meanings of the term 'digital divide' and found that they vary according to how the phenomenon itself is viewed. If the intended meaning calls for the image of physical prowess and willingness of tackle actual problems, then the metaphors of muscle and motion are used. For example, 'bridging' the digital divide conveys the meaning that the divide can be crossed by constructing bridges. On the other hand, when the emphasis is on deeply held values that bind members of a society together, a different type of metaphors is used, such as to 'level the playing field' or to 'connecting to opportunities'. This shows, prima facie, the attitudes toward the digital divide phenomenon that stakeholders, in Courtright's view, do have, but since metaphors usually become frozen with normal use, these attitudes can be imprinted in how words are selected and used. Hence the meaning of 'digital divide' is charged with attitudes and beliefs and refers to some kind of obstacle between two groups of people that needs to be 'bridged' and 'connected.'

${ }^{3}$ Arquette (2002) also traces the intellectual root of talks or discourses about the digital divide and finds that the talks revolve around the 'triumvirate' of development, globalization and society. Talking about the digital divide has become prevalent in development discourses, where the divide is regarded as yet another hurdle against development. It also figures rather prominently in talks about globalization, as it quite dramatically shows the inequalities among nations and cultures. Lastly, digital divide talks play a role in attempts to delineate the information society, thus putting them in the ongoing stream of scholarship in science, technology and society studies (STS). This shows that the digital divide is a social phenomenon. As an object of attention in discourses on development, globalization and society, talks about the digital divide show that the concept is deeply engaged with social scientific studies, and such talks do not seem to be able to distance themselves from talks in areas such as development, globalization, or society. In either case, it seems to show that talks about the digital divide cannot take place meaningfully outside the contexts of its relation to social and historical contexts.

${ }^{4}$ While there is a tremendous amount of information on the digital divide in the West, especially the US, information and research on the phenomenon Asia except Japan and the other Third World countries has attracted far less attention. The figures that most dramatically highlight the disparities between the West and the rest of the world can be found, inter alia, in the Information and Communication Technology (ICT) Development Indices (UNCTAD, 2002). Here the ranking of ICT diffusion by countries, according to the statistics in 2001, shows that the first twenty top ranked countries in 
ICT diffusion are all Western countries, with the US at the top. Only three Asian countries make it to the list: Japan (10), Hong Kong (9) and Singapore (14), whereas among the twenty bottom ranked countries, six are in Africa, and the rest are all in Asia and the Pacific islands (UNCTAD, 2002, p. 35). Thailand, for example, has only 2.27 million people online, or $5.64 \%$ of the whole population (Mekhopee, 2002, p. 150); Japan, on the other hand, has according to an estimate by her Ministry of Post and Telecommunication s more than 56 million, or around $44 \%$ of the population (NUA Internet How Many Online, 2003).

As for the disparities within countries, Karsten Giese has documented the digital divide in China (Giese, 2002) and found that use of the Internet is concentrated on the coast and the big cities such as Beijing, Shanghai as well as Hong Kong and vicinities. World wide web users in these areas account for a staggering $60 \%$, whereas the inland area has only $10 \%$ (Giese 2002, p. 50). In Thailand, $16 \%$ of population in the Bangkok Metropolitan Area have access to the Internet, while only 4-5\% of the people in the rural areas outside of Bangkok do so (Mephokee, 2002, p. 150).

${ }^{5}$ Floridi is keen to point out that action that merely enhances the infosphere is not necessarily better than another action that does not do so but is obviously one that ought to be done. His example is that saving a file, thus putting more information to the infosphere, is not to be preferred over saving a human life (Floridi, pers. comm.). According to him, entropy is an evil since the term refers to the damage of the infosphere, rather than the usual physical property in thermodynamics. However, one is tempted to ask whether there is any theory that could specify which action ought to be preferred when it involves both damaging or promoting the infosphere and the other aspects of reality. Suppose one is faced with a dilemma: Either one has to do A or B. If one chooses to do A, then the decision would enhance, say, some kind of well being of something outside of infosphere, but does nothing to promote or damage it. But if one chooses to do B, then the infosphere would be enhanced, but the kind of well being that would have been promoted had one chosen to do A instead would be lost. The question concerns how in Floridi's theory one could find out which course of action one ought to take.

${ }^{6}$ One may argue that simply having information, whether natural, cultural or technological in Borgmann's term, does not automatically qualify one to be information rich, or to be simply rich in the usual material sense. This is because the possessed information might lack economic value that is derived from the fact that the information is a scarce resource that is wanted by a sufficient number of people having the means to buy it. One may be in possession of a vast amount of information, but if that information is not wanted on the market, then it is as good as nothing, at least in economic terms. However, what I have been saying is that the digital divide problem should be solved not by pouring information from one side of the divide to the other (talking here with the oversimplification), but rather by an effective means of information sharing. As one can be 'rich' in other sense than the economic one, so can one who possesses an amount of information which is valuable in other sense rather than the economic one be rich too.

${ }^{7}$ Floridi, in personal communication, indicated to me that the term 'information' in his theory refers to information objects, not semantic content nor listing of any kind (Floridi, 2003b; see also his article on information in the Blackwell Guide to Philosophy of Computing and Information, available online at http://www.wolfson.ox.ac.uk/ floridi/blackwell/index.htm). But a theory predicated on the premise that ethical norms are based on the size or number of information objects (that constitute the infosphere) would still need an account of semantic representation of those information objects, such as a system of listing or directory, in order that those objects can be accessed and retrieved and thus become usable. De Soto's contribution to development theory, to be discussed in detail in the next section, is precisely that he recognizes the importance of listing and retrieving system for economic development. This should also become more relevant in deliberations on global information justice. ${ }^{8}$ One aspect of philosophy of information that has so far received very little attention is the problem of realism/anti-realism regarding information. Realism regarding information would be a position that information is there objectively in reality (and whether one can have a direct access to it or through any system of representation would be another matter), and anti-realism would hold that 
information would ultimately depend on the mind or the cognitive system of the subject for their being. Given the complex nature of information and the close relationship that it has on the mind and the cognitive system, this is rather surprising.

${ }^{9}$ In fact the role that power plays in the digital divide is investigated in Moss (2002).

${ }^{10}$ Research for this paper has been made possible by two grants from the Thailand Research Fund, No. BRG/14/2544 and No. BRG4680020. I would like to thank Luciano Floridi and an anonymous reviewer for this journal for their valuable comments and suggestions, which saved me from many errors and which led to many improvements.

\section{References}

Arquette, T.J. (2002), 'Social Discourse, Scientific Method, and the Digital Divide: Using the Information Intelligence Quotient (IIQ) to Generate a Multi-Layered Empirical Analysis of Digital Division', Unpublished Ph.D. Thesis, Northwestern University, p. 9. Available at http://www.sla.purdue.edu/people/comm/arquette/dissertation.htm (retrieved January 27, 2003).

Borgmann, A. (1999), Holding on to Reality: The Nature of Information at the Turn of the Millennium, Chicago: University of Chicago Press.

Courtright, C. and Robbin A. (2002), 'Deconstructing the Digital Divide in the United States: An Interpretive Policy Analytic Perspective', paper prepared for International Association of Media and Communication Research and International Communication Association 'Symposium on the Digital Divide,'15-17 November, 2001, Austin, TX, USA.

Floridi, L. (1999), 'Information Ethics: On the Philosophical Foundation of Computer Ethics', Ethics and Information Technology 1, pp. 37-56.

Floridi, L. (2001), 'Ethics in the Infosphere', The Philosophers' Magazine 6, pp. 18-19.

Floridi, L. (2002a), 'What is the Philosophy of Information?' Metaphilosophy 33, pp. 123-145.

Floridi, L. (2002b), 'Information Ethics: An Environmental Approach to the Digital Divide', Philosophy in the Contemporary World 9, pp. 39-45.

Floridi, L. (2003), 'On the Intrinsic Value of Information Objects and the Infosphere', Ethics and Information Technology 4, pp. 287-304.

Floridi, L. and J. W. S. (1999), 'Entropy as Evil in Information Ethics', Etica \& Politica, Special Issue on Computer Ethics, I.2, Oxford University, Computing Laboratory, Programming Research Group Technical Report TR-5-00, available at http://www.univ.trieste.it/ $\sim$ dipfilo/etica_epolitica/1999_2/floridi/e3.html.

Floridi, L. and J. W. S. (2002), 'Computer Ethics: Mapping the Foundationalist Debate', Ethics and Information Technology 4, pp. 1-9.

Giese, K. (2002), 'Wer hat Zugang zum Internet? www-Nutzung und digital divide in China', in Günter Schucher (Hrsg.), Asien und das Internet, Hamburg: Mitteilungen des Instituts für Asienkunde, Nr. 351, pp. 35-62.

Hargittai, E. (2002), 'Second-Level Digital Divide: Differences in People's Online Skills', First Monday, 7, available at http://firstmonday.org/issues/issue7_4/hargittai/index.html

Hongladarom, S. (2001), 'Cultures and Global Justice', Polylog: Forum for Intercultural Philosophizing, 2, pp. 1-34, available at http://www.polylog.org/them/2/fcs2-en.htm.

Mephokee, C. (2002), 'Information Technology: Some Implications for Thailand', in Mitsuhiro Kagami and Masatsugu Tsuji, eds., Digital Divide or Digital Jump: Beyond 'IT' Revolution, Chiba: Institute of Developing Economies, JETRO, pp. 140-167.

Moss, J. (2002), 'Power and the Digital Divide', Ethics and Information Technology 4, pp. 159-165

NUA Internet How Many Online (2003), available at http://www.nua.ie/surveys/how_many_online/ asia.html (retrieved on February 12, 2003).

O'Neill, O. (2000), Bounds of Justice, Cambridge: Cambridge University Press.

Searle, J.R. (1995), The Construction of Social Reality, Penguin Books. 
Smith, M.M. (2001), 'Global Information Justice', Library Trends 49, pp. 519-537.

Soto, H. de (2000), The Mystery of Capital: Why Capitalism Triumphs in the West and Fails Everywhere Else, New York: Basic Books.

United Nations Conference on Trade and Development (2002), Information and Communication Technology (ICT) Development Indices, International Conference on Science, Technology and Innovation: Emerging International Policy Issues, Harvard, 23-24 September 2002. 